\title{
Combinational Effects of Polymer Viscoelasticity and Immobilized Peptides on Cell Adhesion to Cell-selective Scaffolds
}

\author{
Rio Kurimoto, ${ }^{* 1, * 2}$ Kei KanIE, ${ }^{* 3}$ Koichiro Uto, ${ }^{* 2}$ Shun KawaI, ${ }^{* 3}$ Mitsuo Hara, ${ }^{* 4}$ Shusaku Nagano,*5 \\ Yuji NaRITA, ${ }^{* 6}$ Hiroyuki Honda, ${ }^{* 7}$ Masanobu NaITo, ${ }^{* 1, * 8}$ Mitsuhiro Ebara, ${ }^{* 1, * 2, * 9}$ and Ryuji Kato ${ }^{* 3 \dagger}$ \\ *1 Graduate School of Pure and Applied Sciences, University of Tsukuba, 1-1-1 Tennodai, Tsukuba, \\ Ibaraki 305-8577, Japan \\ *2 International Center for Materials Nanoarchitectonics (WPI-MANA), National Institute for Materials \\ Science (NIMS), 1-1 Namiki, Tsukuba, Ibaraki 305-0044, Japan \\ *3 Department of Basic Medicinal Sciences, Graduate School of Pharmaceutical Sciences, Nagoya University, \\ Furo-cho, Chikusa, Nagoya, Aichi 464-8601, Japan \\ *4 Department of Molecular Design and Engineering, Graduate School of Engineering, Nagoya University, \\ Furo-cho, Chikusa, Nagoya, Aichi 464-8603, Japan \\ *5 Nagoya University Venture Business Laboratory, Nagoya University, Furo-cho, Chikusa, Nagoya, \\ Aichi 464-8603, Japan \\ *6 Department of Cardiac Surgery, Nagoya University Graduate School of Medicine, 65 Turumai-cho, Showa, \\ Nagoya, Aichi 466-8550, Japan \\ *7 Department of Biotechnology, Graduate School of Engineering, Nagoya University, Furo-cho, Chikusa, \\ Nagoya, Aichi 464-8603, Japan \\ *8 Structural Materials Unit, Research Center for Strategic Materials, NIMS, 1-2-1 Sengen, Tsukuba, \\ Ibaraki 305-0047, Japan \\ *9 Graduate School of Industrial Science and Technology, Tokyo University of Science, 6-3-1 Niijuku, \\ Katsushika, Tokyo 125-8585, Japan
}

\begin{abstract}
Immobilization of functional peptides on polymer material is necessary to produce cell-selective scaffolds. However, the expected effects of peptide immobilization differ considerably according to the properties of selected polymers. To understand such combinational effects of peptides and polymers, varieties of scaffolds including a combination of six types of poly( $\varepsilon$-caprolactone-co-D,L-lactide) and four types of cell-selective adhesion peptides were fabricated and compared. On each scaffold, the scaffold properties (i.e. mechanical) and their biological functions (i.e. fibroblast- $/$ endothelial cell-/smooth muscle cell-selective adhesion) were measured and compared. The results showed that the cell adhesion performances of the peptides were considerably enhanced or inhibited by the combination of peptide and polymer properties. In the present study, we illustrated the combinational property effects of peptides and polymers using multi-parametric analyses. We provided an example of determining the best scaffold performance for tissue-engineered medical devices based on quantitative data-driven analyses.
\end{abstract}

Keywords Biodegradable polymer, cell adhesion peptide, physicochemical property, viscoelastic property, cell-selective adhesion

(Received July 11, 2016; Accepted September 8, 2016; Published November 10, 2016)

\section{Introduction}

Tissue-engineered vascular grafts (TEVGs), which can regenerate native vascular tissue after implantation, have been studied extensively. ${ }^{1,2}$ To develop TEVGs, biodegradable materials such as poly( $\varepsilon$-caprolactone) (PCL), poly(glycolic acid), and poly(lactic acid) have been used in clinical research. ${ }^{1,2}$ To enhance cell adhesion performance for higher bio-compatibility, scaffolds have been coated with biomolecules such as

† To whom correspondence should be addressed.

E-mail: kato-r@ps.nagoya-u.ac.jp extracellular matrix (ECM) proteins ${ }^{3}$ antibodies $^{4}$ and peptides ${ }^{5-7}$ to improve their in vivo regeneration performance.

Among biomolecules, peptides can be mass-produced in a relatively pure form at low cost. Peptides can control cell adhesion, which is a first step to regenerate tissue. Practically, the control of cell adhesion toward many cell types is important for enhancing regeneration. For example, in artificial blood vessels, the controlled cell adhesion of endothelial cells (ECs) on the inner surface enhances endothelialization, and prevents thrombosis and neointimal hyperplasia. ${ }^{8}$ We have reported several cell-selective adhesion peptides, which can promote selective adhesion of target cells. For example, the Cys-Ala-Gly peptide from ECM enhances EC adhesion but inhibits smooth 


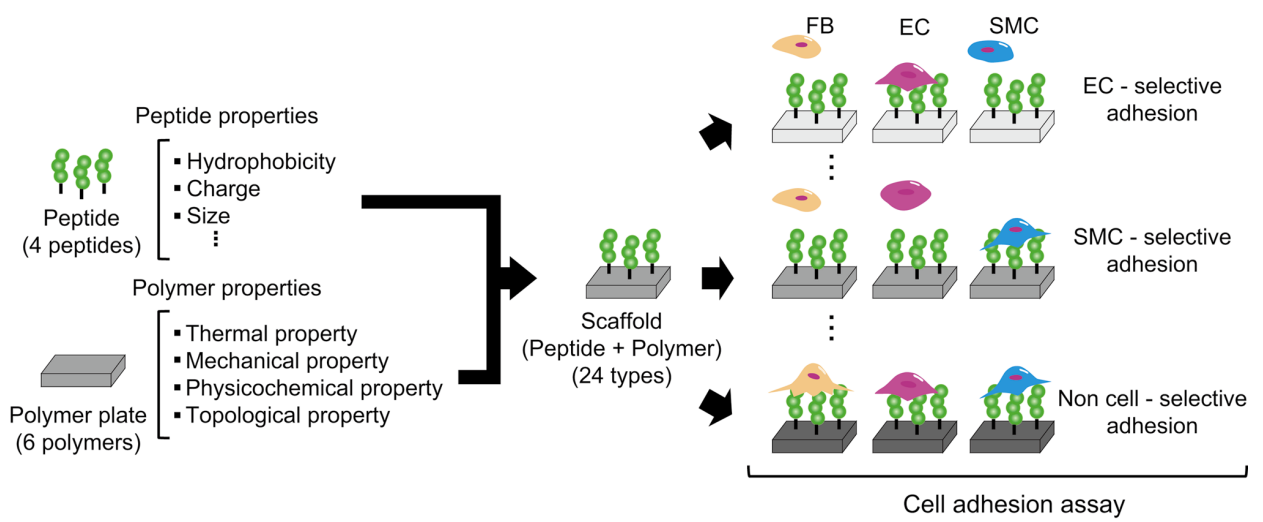

Fig. 1 Schematic image of this study. Cell adhesion performance was evaluated on the scaffolds (peptides + polymer) as a combinational effect of peptides and their immobilized polymer properties. Six types of poly(CL-co-DLLA) plates were fabricated and characterized. The plates were immobilized with four types of peptides. Cell adhesion was evaluated with three types of cells (FBs, ECs, and SMCs) to investigate cell-selective adhesion generated by the combinational effects of polymer properties and peptide properties.

muscle cell (SMC) adhesion. ${ }^{9-11}$ In our study, apart from ECMderived short peptides for cell-selective peptides, we found that several simple peptide sequences also show cell-selective adhesion performances. For example, Ile-repeating peptides show clear EC-selective adhesion capacity, ${ }^{12}$ and various "analog sequences" have shown similar performances. Hence, we propose that there are peptides that can control cell-selective adhesion by their physicochemical property-based effects (e.g. hydrophobicity, electricity, etc.), and not by strict sequencebased effects.

In biomaterial design, the focus has been on the physical and chemical properties of polymers that could affect cell behavior. Recently, the effects of topological and mechanical properties of polymers have been studied as mechanobiology. For example, topological ${ }^{13,14}$ and mechanical ${ }^{15}$ properties could control cell fates and differentiation. We previously reported that viscoelastic and thermal properties of polymers affect cell adhesion and senescence. ${ }^{16,17}$

To develop highly functionalized biomaterials, it is important to investigate the combinational effects of biomolecule properties and their immobilized polymer properties. We reported that cell-selective adhesion is significantly influenced by combining the physicochemical properties of peptides and polymers. ${ }^{18}$ To further enhance the understanding of the combinational effects on cell-selective adhesion, the combinational effect of cell-selective adhesion peptides on viscoelastic and biodegradable scaffolds was investigated in the present study. We fabricated six types of $\operatorname{poly}(\varepsilon$-caprolactoneco-D,L-lactide) (poly(CL-co-DLLA)) plates with a variety of thermal, mechanical, physicochemical, and topological properties by changing the CL/DLLA ratio and unit numbers. On each type of polymer plate, four types of cell-selective adhesion peptides (3-mer) were immobilized, and their performances were compared. To evaluate the cell-selective adhesion on the scaffolds, three types of cells, including fibroblasts (FBs), ECs, and SMCs, were tested for the scaffold application to TEVG (Fig. 1). To understand the complicated relations between peptide and polymer properties, the results of scaffold assays were analyzed by multi-parametric analysis, and visualized using a bubble chart map. The mapped data suggested that there are several factors that can enhance cell-selective adhesion by the combination of polymer and peptide properties.

\section{Material and Methods}

\section{Characterization of poly $(C L-c o-D L L A)$}

Four-armed copolymers with different CL (Tokyo Chemical Industry, Tokyo, Japan) and DLLA (kindly supplied by the Musashino Chemical Laboratory, Tokyo, Japan) compositions were synthesized according to our previous reports. ${ }^{16,17,19}$ Six types of poly(CL-co-DLLA) were characterized using the following measurements (Table 1): Thermal properties of poly(CL-co-DLLA) samples were characterized by DSC (DSC6100, Seiko Instruments, Chiba, Japan). Viscoelastic properties of poly(CL-co-DLLA) samples were tested at $37^{\circ} \mathrm{C}$ using a rheometer (MCR 301, Anton Paar, Tokyo, Japan) with parallel plate geometry (rotating top plate of $25 \mathrm{~mm}$ diameter). The loss tangent and $E$ value were calculated according to previous reports. ${ }^{16,20}$ The contact angle was measured with spin-coated scaffolds of each polymer by FACE CA-XP (Kyowa Interface Science, Saitama, Japan). Roughness profiles of poly(CL-co-DLLA) substrates were examined using a whitelight interferometric microscope (BW-S501, Nikon Instruments, Tokyo, Japan)

\section{Preparation of scaffolds: poly(CL-co-DLLA) immobilized with peptides}

Polymer plates were prepared by the spin-coating method on a round cover glass $(\varphi 13)$ (Matsunami Glass Industry, Tokyo, Japan) with 30 (for 500 unit) and 50 (for 100 unit) wt $\%$ solutions of poly(CL-co-DLLA) using a spin coater (MS-A100, Mikasa Co., Ltd., Tokyo, Japan). To evaporate the solvent left in the membrane, the spin-coated substrates were incubated overnight at $60^{\circ} \mathrm{C}$ in a vacuum. In the present study, the abundance ratios (CL:DLLA) of the polymer plate were defined as 60:40 (100 unit) for P1, 60:40 (500 unit) for P2, 70:30 (100 unit) for P3, 70:30 (500 unit) for P4, 80:20 (100 unit) for P5, and 80:20 (500 unit) for P6.

The immobilization of peptides included two steps. ${ }^{21}$ In the first step, a solution of sulfosuccinimidyl 6-(4'-azido-2'nitrophenylamino) hexanoate (sulfo-SANPAH) (Thermo Fisher Scientific, Kanagawa, Japan) in degassed pure water was prepared at $2.5 \mathrm{mg} \mathrm{ml}^{-1}$. The freshly prepared sulfo-SANPAH solution was placed onto a polystyrene container that the 
Table 1 Characterization of the polymer plates

\begin{tabular}{|c|c|c|c|c|c|c|c|c|c|c|c|c|c|}
\hline \multirow{2}{*}{\multicolumn{3}{|c|}{ Polymer contents in feed }} & \multicolumn{11}{|c|}{ Parameters of polymer plates } \\
\hline & & & \multicolumn{2}{|c|}{$\begin{array}{l}\text { Thermal } \\
\text { property }\end{array}$} & \multicolumn{4}{|c|}{ Mechanical property } & \multirow{2}{*}{$\begin{array}{c}\begin{array}{c}\text { Physicochemical } \\
\text { property }\end{array} \\
\begin{array}{c}\text { Contact } \\
\text { angle } /^{\circ}\end{array}\end{array}$} & \multirow{2}{*}{ 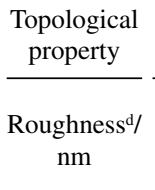 } & \multicolumn{3}{|c|}{$\begin{array}{l}\text { Potential of immobilizing } \\
\text { peptide }\end{array}$} \\
\hline $\begin{array}{l}\text { Sample } \\
\text { name }\end{array}$ & CL:DLLA & $\begin{array}{l}\text { Unit per } \\
\text { branch }\end{array}$ & $\begin{array}{c}\text { Melting } \\
\text { point } \mathrm{a} / \\
{ }^{\circ} \mathrm{C}\end{array}$ & $\begin{array}{c}\text { Delta } \\
\mathrm{H}^{\mathrm{a} /} \\
\mathrm{mJ} \mathrm{mg}^{-1}\end{array}$ & $\begin{array}{c}\text { Storage } \\
\text { modulus } / \\
\mathrm{Pa}\end{array}$ & $\begin{array}{c}\text { Loss } \\
\text { modulus } / \\
\mathrm{Pa}\end{array}$ & $\begin{array}{c}E \text { value }^{\mathrm{b}} / \\
\mathrm{Pa}\end{array}$ & $\begin{array}{c}\text { Loss } \\
\text { tangent } / \text { - }\end{array}$ & & & $\begin{array}{l}\text { Sulfur/ } \\
\text { carbon } \\
\text { ratio } /-\end{array}$ & $\begin{array}{l}\text { Nitrogen/ } \\
\text { carbon } \\
\text { ratio } /-\end{array}$ & $\begin{array}{l}\text { Oxygen/ } \\
\text { carbon } \\
\text { ratio } /-\end{array}$ \\
\hline P1 & $60: 40$ & 100 & 33.8 & 5 & 9.07 & $1.09 \times 10^{3}$ & $3.02 \times 10^{3}$ & 120.18 & 90.2 & 2196 & 0.024 & 0.082 & 0.292 \\
\hline P2 & & 500 & 39.1 & 26 & $3.12 \times 10^{4}$ & $4.13 \times 10^{4}$ & $1.43 \times 10^{5}$ & 1.32 & 94.9 & 133 & 0.009 & 0.032 & 0.264 \\
\hline P3 & $70: 30$ & 100 & 36.9 & 32 & 1.81 & $3.23 \times 10^{2}$ & $8.95 \times 10^{2}$ & 178.45 & 92.8 & 676 & 0.023 & 0.088 & 0.242 \\
\hline P4 & & 500 & 40.5 & 32 & $1.06 \times 10^{4}$ & $2.45 \times 10^{4}$ & $7.39 \times 10^{4}$ & 2.31 & 90.3 & 433 & 0.010 & 0.048 & 0.228 \\
\hline P5 & $80: 20$ & 100 & 45.2 & 47 & $1.72 \times 10^{6}$ & $2.39 \times 10^{5}$ & $4.81 \times 10^{6}$ & 0.14 & 93.4 & 605 & 0.031 & 0.099 & 0.234 \\
\hline P6 & & 500 & 45.7 & 38 & $1.60 \times 10^{7}$ & $1.48 \times 10^{6}$ & $4.45 \times 10^{7}$ & 0.09 & 65.4 & 156 & 0.024 & 0.089 & 0.204 \\
\hline
\end{tabular}

a. Determined by DSC (temperature: $0-120^{\circ} \mathrm{C}$, heating rate: $5^{\circ} \mathrm{C} \mathrm{min}^{-1}$ ). b. Determined by a rheometer (frequency: $0.1-100 \mathrm{rad} \mathrm{s}^{-1}$, strain: $0.1 \%$, temperature: $37^{\circ} \mathrm{C}$ ). c. Measurement was carried out three times (temperature: $37^{\circ} \mathrm{C}$ ). d. The surface roughness was determined from each of the profiles obtained by a white light interferometric microscope. e. Calculated from surface compositions obtained by an X-ray photoelectron spectrometer.

polymer plates were placed on, followed by exposure to UV light (wavelength: $365 \mathrm{~nm}$; intensity: $2.0 \mathrm{~mW} \mathrm{~cm}^{-2}$ ) for $2 \mathrm{~h}$. Then, the polymer plates were washed with pure water three times and immersed in pure water overnight. After washing, the polymer plates were dried in a vacuum. In the second step, a solution of 4-(4,6-dimethoxy-1,3,5-triazin-2-yl)-4-methylmorpholinium chloride n-hydrate (DMT-MM) (Wako Pure Chemical Industries) and peptide in phosphate-buffered saline (PBS) (Wako Pure Chemical Industries) was prepared at 12 and $2 \mathrm{mM}$, respectively. The freshly prepared DMT-MM solution was placed onto a polystyrene container that the polymer plates prepared in the first step were placed on and incubated for $2 \mathrm{~h}$ at room temperature. After $2 \mathrm{~h}$ incubation, the peptide solution (equal volume of DMT-MM solution) was added into the polystyrene container and incubated for $22 \mathrm{~h}$ at room temperature. After incubation, the polymer plates were washed with PBS and pure water three times and stored in a vacuum container until use in the cell adhesion assay. The Arg-GlyAsp-Ser (RGDS) and Met-Met (MM) (>98\% purity) peptides were purchased from the Peptide Institute (Osaka, Japan) and the Gly-Cys-Ala-Gly (CAG), Gly-Ala-Thr-Lys (ATK), Gly-Ile-Ile-Ile (III), and Gly-Arg-Arg-Arg (RRR) peptides (>90\% purity) were synthesized by GL Biochem (Shanghai, China).

To confirm the peptide immobilization performance of prepared polymer plates, the surface compositions on the polymer plates (prepared with $5 \mathrm{wt} \%$ solution) immobilized with MM were measured by X-ray photoelectron spectroscopy (JPS-9000MC/SpecXPS, JEOL, Tokyo, Japan). The composition ratios of oxygen, nitrogen, and sulfur to carbon were each calculated from surface compositions (Fig. S1, Supporting Information).

\section{Cell culture}

Normal human umbilical vein-derived ECs (Kurabo, Osaka, Japan), human aorta SMCs (Kurabo), and normal human adult dermal FBs (Kurabo) were maintained using appropriate culturing methods as previously reported. ${ }^{18}$

\section{Cell adhesion assay}

The cell adhesion assay was carried out on the polymer plates and tissue culture polystyrene (TCPS) as the control. Viable cells stained with Calcein-AM (Dojindo Laboratories, Kumamoto, Japan) for $1.5 \mathrm{~h}$ were seeded on the polymer plate $\left(2.0 \times 10^{4}\right.$ cells cm-2). After cell adhesion at $37^{\circ} \mathrm{C}$ for $1 \mathrm{~d}$, and after gentle PBS wash (3 times), cellular images were obtained on the polymer plate by fluorescence microscopy (X71, Olympus Corporation, Tokyo, Japan) using MetaMorph (Molecular Devices, LLC., Sunnyvale, CA, USA) controlled by an xyz electric stage. Fluorescent cellular images $(N=6)$ were processed using MorphIQ (Ver. 3.10, DITECT, Tokyo, Japan) to count the cellular numbers. To compare the data of cell adhesion from different scaffolds, we first normalized the data within each cell type (FB, EC, or SMC). The above acquired adherent cell numbers from their images normalized by the equation (Eq. (1)) shown below. $X$ is the number of adherent cells, $\mu$ and $\sigma$ are the mean and standard deviation of adherent cell numbers among all samples in each cell type. Then, to further normalize the above normalized cell adhesion rate in each cell type, $Z_{\text {min }}$ (the smallest value in all the $Z$ values among three types of cells) was subtracted from all the data for comparing the data between cell types. We adopted the value of "normalized SMC adhesion rate" on P1 polymer plate as $Z_{\min }$. We designated this value as "normalized cell adhesion rate".

$$
Z=\frac{(X-\mu)}{\sigma}-\left(Z_{\min }\right)
$$

The results of cell adhesion assay were expressed as the mean and standard deviation of the normalized cell adhesion rate. The "proportion of cell adhesion" on cell-selective adhesion peptides immobilized on each polymer plate was obtained by calculating the proportion of "normalized cell adhesion rates" from three types of cells. The significance of the differences between two conditions was tested by Student's t-tests, and the significance of the differences among other conditions was tested by one-way analysis of variance (ANOVA).

\section{Multi-parametric analysis}

Parameter selection to construct models for predicting FB-/EC-/SMC-selective adhesion results was performed by the LASSO logistic regression. Model construction and calculation of the prediction accuracy were described in the previous study. ${ }^{22,23}$ A principle component analysis (PCA) was performed for constructing the map. Principle components 1 and 2 (PC1 and 2) were composed of selected parameters. All analyses were performed using $\mathrm{R}$ software ( $\mathrm{R}$ Development Core Team, https://www.r-project.org/) and the $\mathrm{R}$ package 
(a)

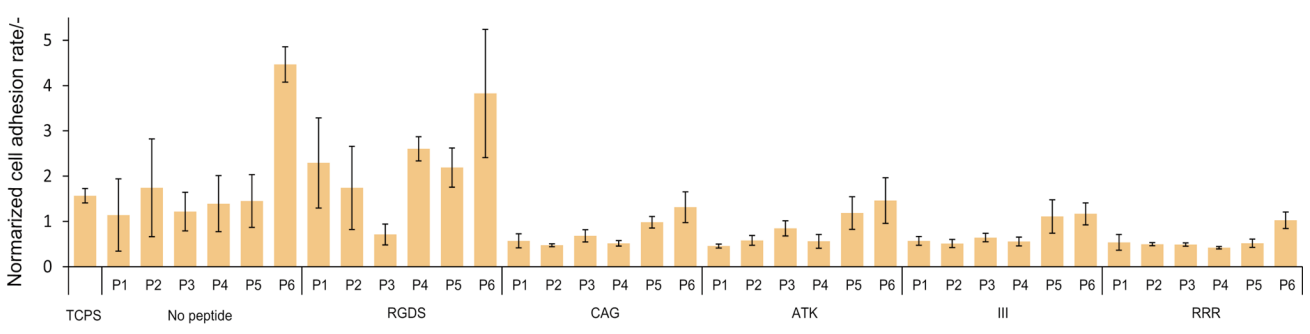

(b)

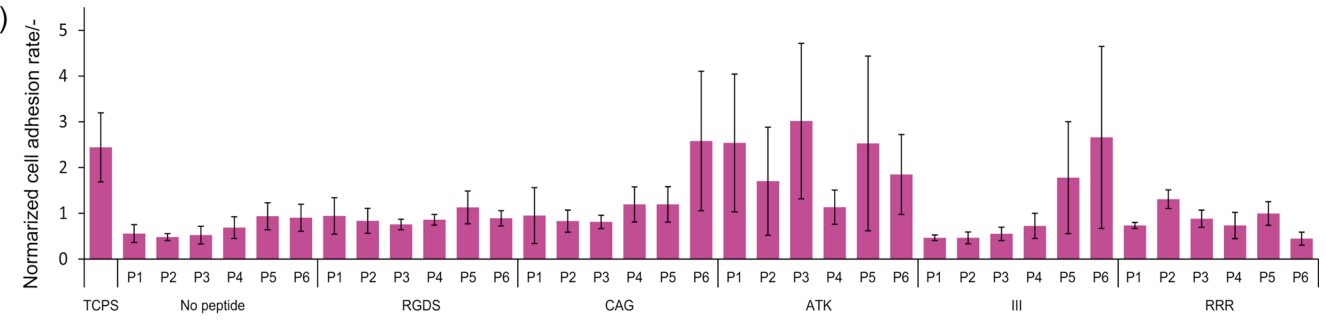

(c)

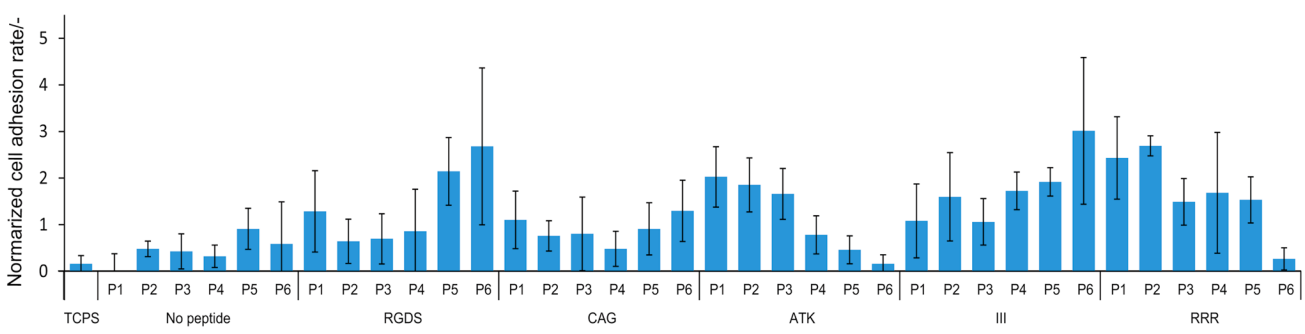

(d)

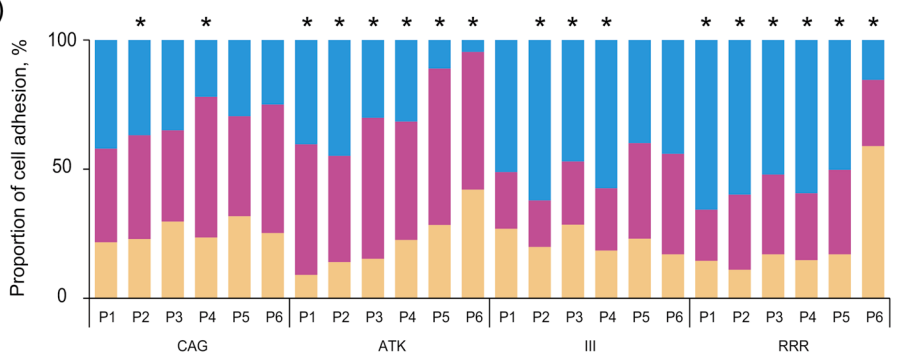

Fig. 2 Cell adhesion performances on the polymer plates immobilized with or without peptides and TCPS. The normalized cell adhesion rate is the number of adherent cells at 1 day after the seeding on the scaffold, which was first normalized within each cell type (standard normalization), and then normalized between cell types. See detailed definition in Material and Methods section. (a) Cell adhesion performance of FB. (b) Cell adhesion performance of EC. (c) Cell adhesion performance of SMC. (d) Summary of cell adhesion rates in three types of cells. The relative cell adhesion rates corresponding to each scaffold is shown as the proportion. The proportion value was calculated from the normalized cell adhesion rates in $\mathrm{FB}, \mathrm{EC}$, and SMC. The $p$ values were evaluated by one-way ANOVA $(* p<0.05)$.

glmnet. ${ }^{24}$ When the prediction model reached sufficient accuracy, the parameter selection results were used for parameter combination interpretation.

\section{Results}

\section{Characterization of polymer properties}

First, to characterize the six polymer plates quantitatively, eight types of polymer properties (two thermal/four mechanical/ one physicochemical/one topological) were measured (Table 1). The thermal properties of polymer plates (melting point and delta $\mathrm{H}$ ), obtained by DSC, indicated that $\mathrm{P} 1$ and $\mathrm{P} 3$ are liquid at $37^{\circ} \mathrm{C}$ (cell culturing condition) since their melting points were $<37^{\circ} \mathrm{C}$. However, other plates were solid at $37^{\circ} \mathrm{C}$. P1 showed lower transition energy (delta $\mathrm{H}$ ) than other polymer plates, which indicated that $\mathrm{P} 1$ had a more amorphous phase. Therefore, the six polymer plates were confirmed to have a variety of thermal properties at $37^{\circ} \mathrm{C}$.

To investigate the mechanical property of polymer plates (storage modulus, loss modulus, $E$ value, and loss tangent), the angular frequency was measured using a rheometer (Table 1 and Fig. S2, Supporting Information). There were wide variations of the $E$ value from $10^{2}$ to $10^{7} \mathrm{~Pa}$. Moreover, the loss tangent was also used as a parameter indicating viscoelasticity of polymer plates. There was a wide variation of the loss tangent from $10^{-2}$ to $10^{2}$. These data indicate that the six polymer plates had various mechanical properties.

The physicochemical properties of polymer plates were determined by the water-contact angle measurement (Table 1). From the results, the water-contact angle of all polymer plates indicated a hydrophobic surface, but was slightly different 
within $90-96^{\circ}$.

The topological properties of polymer plates were determined by the surface roughness (Table 1). From the results, P1 was the roughest polymer plate and the others were similar.

Therefore, these results indicated that the polymer plates used in this study had a wide variety of polymer properties (thermal, mechanical, physicochemical, and topological). Additionally, all polymer properties had no linear correlation to each other.

\section{Effects of polymer properties on cell adhesion}

To investigate the cell adhesive potential of polymer plates, a cell adhesion assay was conducted with three types of cells (FBs, ECs, and SMCs) (Fig. 2 and Figs. S3 - S5, Supporting Information). Figures S3 - S5 show cell adhesion as three types of cells. The images $(N=6)$ were quantified by image analysis (Fig. 2). In this investigation, we designate the bare polymer without any peptide modifications as "polymers," and compared the counted cell numbers on polymer plates with TCPS. The results of polymer plates without peptides indicate that FBs adhered significantly on P6 (P6 vs. TCPS, $p<0.01$ ). However, FB adhesion on other polymer plates was similar, or worse, than that on TCPS. FBs did not show spreading morphology, and remained in a round or spheroid-like aggregation, especially on P1 and P3 (Fig. S3, Supporting Information). EC adhesion decreased 3- to 10-fold on all polymer plates versus that on TCPS (the ratio of EC adhesion on each polymer plate to TCPS). However, SMC adhesion increased up to 3-fold on all polymer plates except for P1 (the ratio of SMC adhesion on each polymer plate to TCPS). These data showed that the cell adhesion of each cell type varied depending on polymer properties. Even with the same polymer, some properties were advantageous to some cells, and some properties were extremely negative. Together with the biased preference to such properties among cell types, it was confirmed that cell-selective adhesion conditions were controlled simply by polymer properties.

\section{Effects of scaffold properties on cell-selective adhesion}

To compare cell-selective adhesion preferences among FBs, ECs, and SMCs on the scaffolds, which were defined as "peptide-immobilized polymers" in this work, cell adhesion was evaluated and compared (Fig. 2 and Figs. S3 - S5, Supporting Information). In this investigation, we designate the bare "polymer" immobilized with "peptides" as "scaffold," and compared the counted cell numbers on scaffolds (with peptide) and scaffolds (without peptide).

First, the effects of RGDS, a sequence-based cell-adhesion peptide, were compared among FBs, ECs, and SMCs for each scaffold. Although FB and SMC adhesion increased on most of the RGDS-immobilized polymer plates, EC adhesion did not increase. The FB adhesion was inhibited on P3, compared with that on the other scaffolds. These results indicated that the cell adhesion with RGDS could be diminished only on some cell types by the combination of polymer properties. Therefore, although RGDS was not evaluated as a cell-selective peptide, ${ }^{18}$ RGDS showed FB-selectivity by the combination with the P4 polymer property.

Second, the cell adhesive potentials of our cell-selective adhesion peptides, CAG (EC-selective), ATK (SMC-selective, Fig. S6, Supporting Information), III (EC-selective), and RRR (FB-selective)-immobilized polymer plates were investigated (Fig. 2). Figure 2 shows that FB adhesion was inhibited on all cell-selective adhesion peptide-immobilized polymer plates. FB adhesion was significantly inhibited 2- to 22-fold, especially on P1 - P4. Therefore, on those polymer plates, the strong adhesion of FBs could be strongly inhibited by the cell-selective adhesion peptides. Figure 2 also shows that EC adhesion increased 2- to 10-fold on all ATK-immobilized polymer plates except P5 (ATK vs. no peptide: $p<0.05$ ). CAG showed EC adhesion increased 4-fold with the combination with P6 (CAG vs. no peptide: $p<0.05)$. On all RRR-immobilized polymer plates, EC adhesions were weak. Therefore, on our polymers, EC adhesion could not be enhanced by coating commonly known adhesion peptides, RGDS or RRR. However, our cell-selective peptides, such as CAG, ATK, and III, could provide EC adhesion on such polymers. SMC adhesion was also enhanced by most of our cell-selective peptide-immobilized polymer plates (III vs. no peptide on all polymer plates: $p<0.05$; ATK $v s$. no peptide on P1 - P4: $p<0.05$; RRR vs. no peptide on P1 - P5: $p<0.05$ ) as shown in Fig. 2. Although SMC adhesion on all CAGimmobilized polymer plates was similar, SMC adhesion increased 4-fold on III-immobilized P6. ATK and RRR increased SMC adhesion 3- to 10-fold on P1 and P2. Therefore, SMC adhesion increased in most combinations of polymer plates and cell-selective adhesion peptides, and the degree of increase depended on the combinations.

To compare the cell-selective properties among different cell types, the proportion of cell adhesion on each polymer plate was calculated from each normalized cell adhesion rate, and compared (Fig. 2(d)). CAG and III, both screened as EC-selective peptides, ${ }^{9,12}$ indicated that the effects of ECselective adhesion could be increased on CAG-immobilized $\mathrm{P} 2 / \mathrm{P} 4$ (FB versus $\mathrm{EC}$ versus $\mathrm{SMC}: p<0.05)$. RRR, screened as an FB-selective peptide, ${ }^{12}$ indicated that the effects of FBselective adhesion could be enhanced by combination with P6 (FB versus EC versus SMC: $p<0.05$ ). ATK, screened as an SMC-selective peptide, indicated EC-selective adhesion, by a combination with $\mathrm{P} 1 / \mathrm{P} 3$ - P6 (FB versus $\mathrm{EC}$ versus SMC: $p<0.05)$. However, the effects of SMC-selective adhesion could be slightly recovered by combination with $\mathrm{P} 2$ (FB versus EC versus SMC: $p<0.05)$. These results indicate that the effects of cell-selective adhesion peptides could be enhanced/ inhibited/inverted by combinations with its immobilized polymers. Therefore, to produce tissue-engineered scaffolds with cell-selective adhesion peptides such as "property-based functional peptides," it is important to consider the ideal combination with polymer and peptide properties.

\section{Visualization of cellular adhesion affected by scaffold property}

The cell-adhesion assay results of peptide and polymer combinations suggested that the potential of cell-selective adhesion could be greatly affected by the combinations of properties of peptides/polymers. However, it has been difficult to understand or extract the rules of these property combinations because of their complex relations. To understand such complex relations, we attempted to visualize their combinations between polymer and peptide properties. Firstly, to objectively find the combinations of parameters that relate to the cell-selective adhesion performance of the scaffold (peptide and its immobilized polymer), parameters that consisted of scaffold properties were selected: polymer properties (11 parameters that could be measured with the scaffold at either a bare or peptide immobilized state) (Table 1), and peptide properties (13 parameters that were calculated from the amino acid index $)^{12,25}$ (Table S1, Supporting Information). For polymer properties, we included varieties of property measurement data, which were both conventionally measured or newly proposed to evaluate cellular compatibility. For peptide properties, we selected amino acid indices that were independent parameters effectively explaining the biological functions of peptides. ${ }^{12}$

Secondly, to objectively find the combinations of parameters 
that effectively explained scaffold performance (cell-selective adhesion), parameter selection through multi-parametric analysis was conducted to automatically extract the combination rule. From the regression models (FB/EC/SMC adhesion prediction model), some parameters were selected as a combination of properties (Table S2, Supporting Information). From the model accuracy, parameter combinations explained either FB or EC adhesion. In the models for predicting FB-/ EC-/SMC-adhesion, coefficients of models indicate importance of polymer/peptide parameters. In the FB- and EC-adhesion, six property parameters were commonly selected as important parameters (Table S2, Supporting Information). In particular, "loss modulus" exhibited a significantly high contribution to both FB- and EC- adhesion prediction. From polymer properties, loss modulus and contact angle were selected. From peptide properties, a single physicochemical property from each amino acid position was selected. For explaining FB adhesion, melting point and delta $\mathrm{H}$ from polymer properties were included in the parameter combinations. For explaining EC adhesion, the sulfur/carbon ratio from polymer properties was included in the parameter combinations. These results showed that there are complex combinational effects in scaffold performances, which could be explained by both peptide and polymer parameters.

To further interpret such complex combinational effects of peptide and polymer properties on cell-selective adhesion, PCA was performed (Table S3, Supporting Information). From the PCA plot (Fig. S7, Supporting Information), it was found that the property parameters were divided into two groups; PC1 consisted of polymer properties, whereas PC2 consisted of peptide properties. When PCA maps were compared between cell type, each map indicated that there were different causes that affected cell-selective adhesion in each cell types. From Fig. S7(a) (Supporting Information), it can be seen that FBadhesion was less affected by differences in peptide properties (PC2), but more affected by polymer properties (PC1) linearly. From Figs. S7(b) and S7(c) (Supporting Information), it is noted that EC-/SMC-adhesions were more complex. There were localized maximum effects of peptide and polymer combinations at several spots on the map. Figure S7(b) showed that the peptide property to design EC-adhesion could be described to be low in normalized van der Waals volume at the N-terminus first residue, high in free energy in the alpha helical region at the second residue, and high in alpha helix indices for beta proteins at the $\mathrm{C}$-terminus residue to mark a larger PC2. However, even with a lower PC2 than PC1, the polymer property showed a higher value in terms of the sulfur/carbon ratio, loss modulus, and contact angle, and EC adhesion was achieved. From Fig. S7(c), the localized combination spots were as follows: (1) lower PC1 and PC2 scores, (2) lower PC1 and higher PC2 scores, and (3) higher PC1 and medium PC2 scores (III peptide). Considering such property combination maps indicating multiple local effects, it is clear that the scaffold with better function should be carefully designed from both aspects: peptide and polymer properties. The data indicated that multiparametric analyses of measurement data for characterizing the scaffold could be effectively used to determine their design concept.

\section{Discussion}

In the design of biocompatible polymers functionalized with biomolecules, there are several reports indicating a variety of parameters affect cell-polymer interactions. ${ }^{26}$ Polymers not only simply control the biomolecule characteristics, but studies have indicated that polymers have a variety of properties that can enhance cellular responses. To design a scaffold that can maximize the biomolecule function on sophisticatedly designed polymers for tissue engineering applications, there should be more practical and quantitative data analyses to understand the complex combinational effects of parameters derived from both biomolecules and polymers.

Therefore, we previously reported that the cell adhesion performance of the same adhesion peptide could be greatly increased/decreased by changing the wettability of the immobilized smart polymer. ${ }^{18}$ However, recently, it was determined that the physicochemical properties (e.g. wettability) were not only the critical factor changing the scaffold performance. Topological and mechanical properties in the mechanobiological field have been studied extensively in the last few decades. ${ }^{13-15}$ In the present study, we therefore investigated the effects of a variety of polymer properties (thermal, mechanical, physicochemical, and topological) related to cell adhesion. To the best of our knowledge, our findings in the current study provided the first quantitative investigation of the combinational effects of such polymer properties, together with our original functional cell-selective adhesion peptides.

In our polymer plate properties (Table 1), we considered that our fabricated scaffolds covered a variety of peptide and polymer properties to show new aspects. We believe that our detailed data with such a variety of properties shows a property-regulated cellular response, compared with the conventionally known biology-regulated cellular response. For example, our polymer plates have covered the range of loss modulus from $10^{2}$ to $10^{6}$ $\mathrm{Pa}$ (Table 1). This range of the loss modulus has been covered in most previous reports. ${ }^{16,20}$ From these data, a spheroid-like aggregation of FBs on $\mathrm{P} 1$ and $\mathrm{P} 3$ was found, and it matched with the results of NIH3T3 fibroblasts forming aggregations in our previous reports. ${ }^{16}$ Although FB is commonly known as a tough cell that can adhere and grow in various conditions, this result reproduces the FB response to the difference of loss modulus of the scaffold. Surprisingly, such an FB response was also observed on the RGDS-immobilized P3. This suggests that these property effects can overpower the biological effect (i.e. integrin-associated adhesion) in some conditions. It was also interesting that from the parameter selection, loss modulus relating to viscoelasticity, was selected (Table S3, Supporting Information). The water contact angle was an important parameter to induce cellular adhesion. However, our data show that even slight differences of the water contact angle greatly changed cellular adhesion (Tables S2-S3, Supporting Information). In our scaffolds, the cell adhesion of FBs and SMCs increased to a greater extent than that of TCPS, whereas that of ECs decreased. These results suggest that polymers without biomolecules could generate cell selectivity. These findings support our proposed concept that some peptides can control cell-selective adhesion by their property-based functions, rather than their sequence effects. In our previous reports, ${ }^{9,12}$ we confirmed these effects by showing various analog sequences which peptide sequence is not the same, although shows similar functions (i.e. AAA, AAC, and AAG). However, in this work, we extended our investigation by showing that these celladhesion functions could also be designed by artificial polymer properties.

Regarding peptides, our data indicated a trend of peptide and polymer property combinations that maximized peptide performances. The EC-selective adhesion peptide, CAG, was originally obtained from a peptide array screening, and was applied to the PCL polymer resulting in the enhancement of 


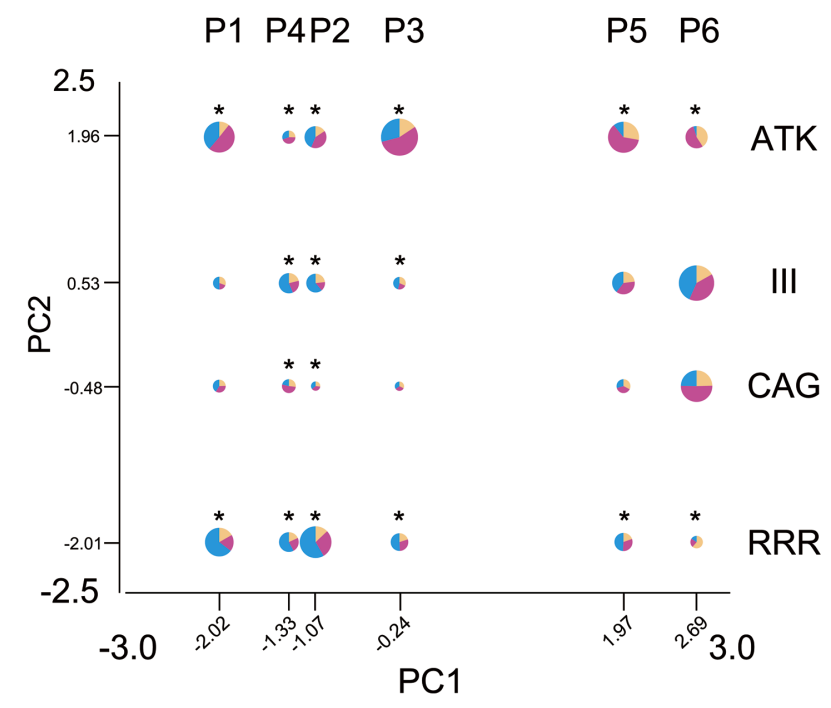

Fig. 3 A cell-selective adhesion map based on scaffold properties (PC1: polymer properties, PC2: peptide properties). The size of each circle was determined by the largest amount of cell adhesion among three types of cells (FBs (yellow), ECs (pink), SMCs (blue)). The proportion values of each cell in the circles were the same as the data for Fig. 2(d). The $p$ values were evaluated by one-way ANOVA $(* p<0.05)$.

EC-selective adhesion in vitro and vivo.9-11 However, among our scaffolds in this work, the CAG provided its EC-selective adhesion performance on P2, P4, and P6. By the fold-change, P2 enhanced EC-adhesion 1.7-fold higher than FB-adhesion, P4 and P6 enhanced EC-adhesion higher than both FB- and SMC-adhesion (>2.0-fold). In other words, on other polymers, the peptide performance was changed. As a comparison, when P2, P4, and P6 were functionalized by RGD peptides, such EC-adhesion was only 0.2 to 1.0 -fold higher than other cells (Table S4, Supporting Information). We consider that such reduction of previously confirmed CAG performance on certain conditions, was owing to the combinational effect of peptide and polymer properties. The ATK (SMC-selective adhesion in Fig. S6, Supporting Information), and RRR (FB-selective adhesion in previous report) ${ }^{12,18}$ also retained their function on some polymers, however changed (or even inversed) the function on other polymers. Also from our previous studies, such combinational effect of peptide and polymer, which change the original peptide function, had been also found with III, another EC-selective adhesion peptide. ${ }^{18}$ This also suggests that this cell-selective adhesion performance was supported by the peptide sequence/polymer itself, and their combinations. Therefore, this shows the risk that some functional peptides obtained from screening on different solid platforms can easily be changed (enhanced or strengthened) by polymer properties, and their optimum condition should be carefully determined by systematic analyses, such as our data.

To design cell-selective scaffolds systematically, the correlation of cell-selective potential of scaffolds and combinations of peptide and polymer properties were visualized as a cell-selective adhesion map in our work (Fig. 3). Such mapping interpretations suggest the importance of active data usage describing biomolecule and polymer properties with a multi-parametric analysis. Construction and accumulation of such peptide and polymer effects databases, and should contribute to a guide for the optimum combination of peptides and polymers for fabrication. Moreover, considering that peptide and polymer properties consisted of different PCA components, such databases could possibly help to predict the best matching peptide/polymer candidate properties to match the technology and resources of fabrication facilities.

\section{Conclusions}

In the design of a cell-selective adhesion function on polymerbased scaffolds for medical applications, our work shows the clear combinational effect of peptide and polymer properties. These findings suggest the importance of analyzing both peptide and polymer property data, since property-based cellular interaction has the potential to provide the cell-selective adhesion performance of scaffolds necessary for medical use. Moreover, the data supports our proposed concept of property as another important trigger in regulating cellular responses. Effective property-based cell regulation mechanisms would result in more cost effective and reproducible medical materials, because scaffold property could then be designed chemically.

\section{Acknowledgements}

This work was supported by JSPS KAKENHI, Grant Number JP 15K21070. Part of this work was supported by the Nanotechnology Platform Program (Molecule and Polymer Synthesis) of the Ministry of Education, Culture, Sports, Science and Technology (MEXT), Japan. R. K. acknowledges the technical support of Y. Kondo, M. Okada, and A. Ooguchi (Nagoya University). We would like to thank Editage (www. editage.jp) for English language editing.

\section{Supporting Information}

Supporting Information includes peptide immobilization performance (Fig. S1) and dynamic viscoelasticity of the polymers (Fig. S2), cellular images (FB, EC, and SMC) (Figs. S3 - S5), SEM images of cell adhesion on PCL membranes immobilized with ATK (Fig. S6), bubble chart maps of each cell adhesion constructed with scaffold properties (Fig. S7), indices of amino acid residues (Table S1), selected parameters as scaffold properties by LASSO logistic regression (Table S2), PCA with selected scaffold properties (Table S3), and EC-selective adhesion performance of CAG and RGDS (Table S4). This material is available free of charge on the Web at http://www.jsac.or.jp/analsci/.

\section{References}

1. G. P. Chen, T. Ushida, and T. Tateishi, J. Biomed. Mater. Res., 2000, 51, 273.

2. T. Yokota, H. Ichikawa, G. Matsumiya, T. Kuratani, T. Sakaguchi, S. Iwai, Y. Shirakawa, K. Torikai, A. Saito, E. Uchimura, N. Kawaguchi, N. Matsuura, and Y. Sawa, J. Thorac. Cardiovasc. Surg., 2008, 136, 900.

3. K. O. Cardinal and S. K. Williams, Tissue Eng. Part A, 2009, 15, 3869.

4. J. Aoki, P. W. Serruys, H. van Beusekom, A. T. L. Ong, E. P. McFadden, G. Sianos, W. J. van der Giessen, E. Regar, P. J. de Feyter, H. R. Davis, S. Rowland, and M. J. B. Kutryk, J. Am. Coll. Cardiol., 2005, 45, 1574. 
5. M. D. Pierschbacher and E. Ruoslahti, Nature, 1984, 309, 30.

6. J. Graf, R. C. Ogle, F. A. Robey, M. Sasaki, G. R. Martin, Y. Yamada, and H. K. Kleinman, Biochemistry, 1987, 26, 6896.

7. M. Yin, Y. Yuan, C. Liu, and J. Wang, Biomaterials, 2009 , 30, 2764.

8. D. Kong, L. G. Melo, A. A. Mangi, L. Zhang, M. LopezIlasaca, M. A. Perrella, C. C. Liew, R. E. Pratt, and V. J. Dzau, Circulation, 2004, 109, 1769.

9. K. Kanie, Y. Narita, Y. Z. Zhao, F. Kuwabara, M. Satake, S. Honda, H. Kaneko, T. Yoshioka, M. Okochi, H. Honda, and R. Kato, Biotechnol. Bioeng., 2012, 109, 1808.

10. M. Khan, J. Yang, C. Shi, J. Lv, Y. Feng, and W. Zhang, Acta Biomater., 2015, 20, 69.

11. F. Kuwabara, Y. Narita, A. Yamawaki-Ogata, K. Kanie, R. Kato, M. Satake, H. Kaneko, H. Oshima, A. Usui, and Y. Ueda, Ann. Thorac. Surg., 2012, 93, 156.

12. K. Kanie, R. Kato, Y. Z. Zhao, Y. Narita, M. Okochi, and H. Honda, J. Pept. Sci., 2011, 17, 479.

13. C. S. Chen, M. Mrksich, S. Huang, G. M. Whitesides, and D. E. Ingber, Science, 1997, 276, 1425.

14. D. E. Ingber, Prog. Biophys. Mol. Biol., 2008, 97, 163.

15. A. J. Engler, S. Sen, H. L. Sweeney, and D. E. Discher, Cell, 2006, 126, 677.
16. K. Uto, S. S. Mano, T. Aoyagi, and M. Ebara, ACS Biomater. Sci. Eng., 2016, 2, 446.

17. S. S. Mano, K. Uto, T. Aoyagi, and M. Ebara, AIMS Mater. Sci., 2016, 3, 66.

18. R. Kurimoto, K. Kanie, N. Idota, M. Hara, S. Nagano, T. Tsukahara, Y. Narita, H. Honda, M. Naito, M. Ebara, and R. Kato, Int. J. Polym. Sci., 2016, 2016, 9.

19. K. Uto, T. Muroya, M. Okamoto, H. Tanaka, T. Murase, M. Ebara, and T. Aoyagi, Sci. Technol. Adv. Mater, 2012, 13.

20. A. R. Cameron, J. E. Frith, and J. J. Cooper-White, Biomaterials, 2011, 32, 5979.

21. B. Li, J. X. Chen, and J. H. C. Wang, J. Biomed. Mater. Res. A, 2006, 79A, 989.

22. H. Sasaki, I. Takeuchi, M. Okada, R. Sawada, K. Kanie, Y Kiyota, H. Honda, and R. Kato, Plos One, 2014, 9.

23. K. Ito, A. Hikida, S. Kawai, L. Vu Thi Tuyet, T. Motoyama, S. Kitagawa, Y. Yoshikawa, R. Kato, and Y. Kawarasaki, Nat. Commun., 2013, 4.

24. J. Friedman, T. Hastie, and R. Tibshirani, J. Stat. Softw., 2010, 33, 1 .

25. S. Kawashima, P. Pokarowski, M. Pokarowska, A. Kolinski, T. Katayama, and M. Kanehisa, Nucleic Acids Res., 2008, 36, D202.

26. K. Ye, X. Wang, L. P. Cao, S. Y. Li, Z. H. Li, L. Yu, and J. D. Ding, Nano Lett., 2015, 15, 4720. 